\title{
Unfolding Concurrent Well-Structured Transition Systems ${ }^{\star}$
}

\author{
Frédéric Herbreteau, Grégoire Sutre, and The Quang Tran \\ LaBRI, CNRS UMR 5800, Domaine Universitaire, Talence, France \\ $\{$ fh, sutre, tran\}@labri.fr
}

\begin{abstract}
Our main objective is to combine partial-order methods with verification techniques for infinite-state systems in order to obtain efficient verification algorithms for concurrent infinite-state systems. Partial-order methods are commonly used in the analysis of finite systems consisting of many parallel components. In this paper we propose an extension of these methods to parallel compositions of infinite-state systems. We argue that it is advantageous to model each component by an event structure as this allows us to exhibit the concurrency present implicitly in some infinite-state systems such as automata with queues or counters. We generalize the notion of complete prefix from 1-safe Petri nets to all well-structured transition systems. We give an on-the-fly unfolding algorithm which given event structures representing the components produces an event structure representing their synchronized product. A prototype implementation demonstrates the benefits of our approach.
\end{abstract}

\section{Introduction}

Partial-order methods [God96, Val91, Pel93] are frequently used for the verification of programs, in particular for systems of concurrent processes. Indeed, proving that the computations of such systems meet some requirement often results in the well-known exponential blow-up due to interleaving of concurrent actions. Partial-order methods tackle this problem by selecting a hopefully small set of relevant computations that are sufficient to conclude. Selecting among the interleavings is sound because ordering concurrent (independent) actions is irrelevant. Hence, instead of considering totally ordered computations, one analyses traces [Maz86] that stand for all equivalent computations w.r.t. concurrency. As a consequence, the whole computation tree can be partially rather than totally ordered resulting in event structures [NPW81, NRT95]. Efficient exploration techniques, e.g. unfolding [McM95, NRT95], exist for partiallyordered structures.

Traditionally, partial-order methods have been applied to concurrent finite-state processes and proved to be successful. In this paper, we apply partial-order methods to parallel compositions of infinite-state systems such as counter machines and communicating finite-state machines. Verification of infinite-state systems is known to be

\footnotetext{
* This work was partially supported by the French Ministry of Research (Project PERSÉE of the ACI Sécurité et Informatique).
} 
undecidable, however some classes of infinite-state systems enjoy nice decidability results. Well-Structured Transition Systems [Fin90, AČJT00, FS01] provide us with a nice framework based on weak simulation relations that are well-preorders. Since a well-preorder admits no infinite decreasing sequence, one can consider only a finite prefix of the computation tree in order to decide properties like boundedness, termination or covering.

Our contribution. Our goal is to analyse synchronized products of well-structured systems using a method similar to Petri net unfolding in order to obtain event structures. The most straightforward approach would be to consider that each component generates an (infinite) transition system, then use an on-the-fly variant of the unfolding method for parallel composition of finite automata. This turns out not to be satisfactory: imagine that one models a counter by an (infinite) automaton then if, say, three different processes want to increase the counter, their actions will get interleaved on the automaton modeling the counter. As in principle those actions are independent, we lose a good deal of concurrency present in the original system. Our solution is to model each component by an event structure, hence taking advantage of the intrinsic concurrency present in each component.

Our first contribution is an algorithm for constructing event structures for components modeling counters. It results in event structures with more concurrency than in [McM95]. We also present a general algorithm that works for all (infinite) transition systems. This is not trivial: one wants the most concurrent event structure whereas concurrency is not explicit in transition systems. Of course, our algorithm is less efficient than dedicated algorithms (e.g. for counter automata), however it exhibits a good deal of concurrency using local independence. For instance it produces the same event structures as those reported in [LI05] for queue automata.

Our second contribution is a generalization of the unfolding method of [ER99] to parallel composition of potentially infinite event structures. Our algorithm is both capable of exploiting concurrency in components as well as among them. In particular we show that modeling Petri nets as the parallel composition of its places (viewed as counters) results in very efficient analysis using our algorithms.

Of course there is no hope to have a notion of complete prefix for a parallel composition of infinite systems. There is hope though when the components are well-structured systems. We give a property-preserving truncation criterion for event structures of well-structured transition systems. The resulting (complete) prefix contains enough information to decide boundedness, termination and quasi-liveness. We also show preservation of well-structure under parallel composition for all variants of well-structure. Remark that these results cannot be directly obtained from previous techniques on wellstructured systems, since the complete prefix is not a compact partial-order representation of the finite reachability tree of [FS01].

Related work. The unfolding technique [McM95] has been developed for several models of concurrency, e.g. synchronous products of transition systems [ER99], high-level Petri nets [KK03], extended finite state machines [LI05], symmetric Petri nets [CGP01]. However, all these techniques deal with finite-state models.

In [AJKP98, the authors address the coverability problem for infinite state systems by combining partial-order reductions and symbolic backward computations. The 
unfolding of unbounded Petri nets was recently considered. In [AIN00] Abdulla et al. propose a backward unfolding technique for coverability analysis, and [DJN04] presents an unfolding-based adaptation of Karp and Miller's algorithm. Our method generalizes these results: it analyses any (infinite) well-structured transition system [FS01], offering both forward and backward approaches, hence enabling to check covering, boundedness and termination properties.

Outline. Section 2 introduces notations and definitions for transition systems and event structures. In section 3 we prove well-structure properties for event structures. Then, in section 4 we introduce our algorithms for unfolding systems. Finally, in section 5 we give some experimental results showing the benefits of our approach, and we conclude on future work. Please note that some preliminary (standard) definitions along with all proofs had to be omitted due to space constraints. A long version of this paper can be obtained from the authors.

\section{Labeled Transition Systems and Event Structures}

A binary relation $R$ on some set $U$ is any subset of $U \times U$. We will sometimes view functions as relations. Given a subset $X \subseteq U$, we denote by $R[X]$ the relational image of $X$ through $R$, defined by $R[X]=\{y \in U / \exists x \in X, x R y\}$. The inverse of $R$ is the binary relation $R^{-1}$ on $U$ defined by $x R^{-1} x^{\prime}$ iff $x^{\prime} R x$. A preorder on some set $U$ is any reflexive and transitive relation $\preceq$ on $U$. We let $x \prec x^{\prime}$ denote $x \preceq x^{\prime} \npreceq x$. Given a preorder $\preceq$ on $U$, the inverse relation $\preceq^{-1}$ is a preorder also written $\succeq$. For any subset $X \subseteq U$, the set $\preceq[X]$ (resp. $\succeq[X]$ ) is called the upward closure (resp. downward closure) of $X$ with respect to $\preceq$. We say that $X$ is upward-closed (resp. downwardclosed) if $X$ is equal to its upward closure (resp. downward closure). A partial order on $U$ is any antisymmetric preorder on $U$. Given a partial order $\leq$ on $U$, a maximal element (resp. minimal element) of some subset $X \subseteq U$ is any $m \in X$ such that $m^{\prime} \nsucceq m$ (resp. $\left.m^{\prime} \not \leq m\right)$ for all $m^{\prime} \neq m$ in $X$. We write $\operatorname{Max}_{\leq}(X)\left(\operatorname{resp} . \operatorname{Min}_{\leq}(X)\right)$ for the set of maximal elements (resp. minimal elements) of $X$ with respect to $\leq$.

Given a set $\Sigma$, we denote by $\Sigma^{*}$ (resp. $\Sigma^{\omega}$ ) the set of all finite (resp. infinite) sequences $a_{1}, a_{2}, \ldots, a_{k}$ (resp. $a_{1}, a_{2}, \ldots, a_{k}, \ldots$ ) of elements in $\Sigma$. The empty sequence is written $\varepsilon$ and we denote by $\Sigma^{+}$the set $\Sigma^{*} \backslash\{\varepsilon\}$.

\subsection{Labeled Transition Systems}

Definition 2.1. A labeled transition system (LTS) is a 4-tuple $\mathcal{S}=\left(S, s^{0}, \Sigma, \rightarrow\right)$ where $S$ is a set of states, $s^{0} \in S$ is an initial state, $\Sigma$ is a set of labels and $\rightarrow \subseteq S \times \Sigma \times S$ is $a$ transition relation.

A transition $\left(s, a, s^{\prime}\right) \in \rightarrow$ is also written $s \stackrel{a}{\rightarrow} s^{\prime}$. We also write $s \stackrel{a}{\rightarrow}$ whenever there exists $s^{\prime}$ such that $s \stackrel{a}{\rightarrow} s^{\prime}$. A finite path (resp. infinite path) in $\mathcal{S}$ is any finite (resp. infinite) sequence $\pi=s_{1} \stackrel{a_{1}}{\longrightarrow} s_{1}^{\prime}, s_{2} \stackrel{a_{2}}{\longrightarrow} s_{2}^{\prime}, \ldots, s_{k} \stackrel{a_{k}}{\longrightarrow} s_{k}^{\prime}, \ldots$ of transitions such that $s_{i-1}^{\prime}=s_{i}$ for every index $i>1$ in the sequence. We shortly write $\pi=s_{1} \stackrel{a_{1}}{\longrightarrow} s_{2} \stackrel{a_{2}}{\longrightarrow}$ $s_{3} \cdots s_{k} \stackrel{a_{k}}{\longrightarrow} s_{k+1} \cdots$ and we say that $\pi$ starts in $s_{1}$. A finite (resp. infinite) execution 
of $\mathcal{S}$ is any finite (resp. infinite) path starting in the initial state $s^{0}$ of $\mathcal{S}$. Slightly abusing notations, we will also write $s \stackrel{\varepsilon}{\rightarrow} s$ for every state $s$. The reachability set post ${ }_{\mathcal{S}}^{*}$ of $\mathcal{S}$ is the set of states that are visited by some execution.

We now present the composition primitive that we use to build complex systems from basic components: the synchronized product of labeled transitions [Arn94]. In a synchronized product, components must behave according to so-called synchronization vectors. Consider $n$ labeled transition systems $\mathcal{S}_{1}, \ldots, \mathcal{S}_{n}$ with $\mathcal{S}_{i}=\left(S_{i}, s_{i}^{0}, \Sigma_{i}, \rightarrow_{i}\right)$. A synchronization vector is any $n$-tuple $\boldsymbol{v}$ in $\Sigma_{\otimes}=\left(\Sigma_{1} \cup\{\varepsilon\}\right) \times \cdots \times\left(\Sigma_{n} \cup\{\varepsilon\}\right)$, and a synchronization constraint is any subset $V \subseteq \Sigma_{\otimes}$ of synchronization vectors. Intuitively, a label $a$ in a synchronization vector means that the corresponding component must take a transition labeled by $a$, whereas an $\varepsilon$ means that the component must not move.

Definition 2.2. The synchronized product of $n$ labeled transition systems $\mathcal{S}_{1}, \ldots, \mathcal{S}_{n}$ with respect to a synchronization constraint $V$ is the labeled transition system $\mathcal{S}_{\otimes}=$ $\left(S_{\otimes}, \boldsymbol{s}_{\otimes}^{\mathbf{0}}, \Sigma_{\otimes}, \rightarrow_{\otimes}\right)$ defined by: $S_{\otimes}=S_{1} \times \cdots \times S_{n}, \boldsymbol{s}^{\mathbf{0}}{ }_{\otimes}=\left\langle s_{1}^{0}, \ldots, s_{n}^{0}\right\rangle$ and $\boldsymbol{s} \stackrel{\boldsymbol{v}}{\rightarrow} \boldsymbol{s}^{\mathbf{\prime}}$ iff $\boldsymbol{v} \in V$ and $\boldsymbol{s}(i) \stackrel{\boldsymbol{v}(i)}{\longrightarrow} \boldsymbol{s}^{\prime}(i)$ for every $1 \leq i \leq n$.

\subsection{Labeled Event Structures}

Definition 2.3. A labeled event structure (LES) is a 5-tuple $\mathcal{E}=(E, \leq, \#, \Sigma, l)$ where $E$ is a set of events, $\leq$ is a partial order on $E$, \# is a symmetric and irreflexive relation on $E, \Sigma$ is a set of labels, and $l: E \rightarrow \Sigma$ is a labeling function satisfying:

i) the downward closure $\geq[\{e\}]$ is finite for every $e \in E$, and

ii) $e \# e^{\prime}$ and $e^{\prime} \leq e^{\prime \prime}$ implies $e \# e^{\prime \prime}$ for every $e, e^{\prime}, e^{\prime \prime} \in E$.

In the previous definition, relations $\leq$ and \# are respectively called causality and conflict relations. Intuitively, an event $e$ can occur when (1) every causal event $e^{\prime}$ with $e^{\prime} \leq e$ has already occurred and (2) no conflicting event $e^{\prime}$ with $e^{\prime} \# e$ has already occurred. Condition $i$ ) enforces that any event has finitely many causal events, and condition $i$ ) expresses a conflict inheritance property.

A subset of $E$ is called conflict-free if it does not contain any two events that are in conflict. A configuration is any conflict-free and downward-closed (w.r.t. causality) subset of $E$. We denote by $\mathcal{C}(\mathcal{E})$ (resp. $\mathcal{C}_{f}(\mathcal{E})$ ) the set of all configurations (resp. finite configurations) of a labeled event structure $\mathcal{E}$. For any event $e \in E$, the set $\geq[\{e\}]$ is called the local configuration of $e$ (it is readily seen that this set is a finite configuration). We will shortly write $[e]$ the local configuration of $e$ when the causality preorder is clear from the context. An event $e \in E$ is enabled at some configuration $C$, written $C \vdash e$, if $e \notin C$ and $C \cup\{e\}$ is a configuration. We say that a labeled event structure is finitelybranching if every finite configuration has finitely many enabled events. A variant of König's lemma applies to finitely-branching labeled event structures.

Definition 2.4. A marking for a labeled event structure $\mathcal{E}$ is any function $M$ from $\mathcal{C}_{f}(\mathcal{E})$ to some set $S$. 
A marked LES is any pair $(\mathcal{E}, M)$ consisting of a labeled event structure $\mathcal{E}$ and a marking $M$ for $\mathcal{E}$. We denote by $\mathcal{S}_{\mathcal{E}}^{M}$ the labeled transition system induced by $(\mathcal{E}, M)$ and defined by $\mathcal{S}_{\mathcal{E}}^{M}=\left(M\left[\mathcal{C}_{f}(\mathcal{E})\right], M(\emptyset), \Sigma, \rightarrow\right)$ where $s \stackrel{a}{\rightarrow} s^{\prime}$ iff there exists a finite configuration $C$ and an event $e$ enabled at $C$ such that $s=M(C), a=l(e)$ and $s^{\prime}=M(C \cup\{e\})$. Given a labeled transition system $\mathcal{S}$, a marked LES for $\mathcal{S}$ is any marked LES $(\mathcal{E}, M)$ such that $\mathcal{S}_{\mathcal{E}}^{M}$ coincides with the restriction of $\mathcal{S}$ to post. ${ }_{\mathcal{S}}^{*}$. Remark that $(\mathcal{E}, M)$ is obviously a marked LES for $\mathcal{S}_{\mathcal{E}}^{M}$.

\section{Truncation for Well-Structured Transition Systems}

Well-Structured Transition Systems were introduced in [Fin90, AČJT00] as an abstract generalization of Petri nets satisfying the same monotonicity property, and hence enjoying nice decidability properties. It turns out that many classes of infinite-state systems are well-structured [FS01].

We will see in the next section how to algorithmically construct labeled event structures. However, a labeled event structure is infinite as soon as the underlying system has an infinite execution. Thus, we need property-preserving truncation techniques in order to decide verification problems using only a finite prefix of an event structure. In this section, we show how such techniques can be obtained when the underlying system is well-structured.

For simplicity we only focus, without loss of generality, on forward analysis techniques for well-structured transition systems. We show in the long version of this paper how known backward analysis results on well-structured transition systems can be captured by this forward analysis. Moreover, we do not discuss effectivity issues (such as whether preorders need to be decidable, whether successor states need to be computable, etc.) since they are basically the same as in [FS01].

\subsection{Synchronized Product of Well-Structured Transition Systems}

Recall that our main objective is to verify complex systems obtained by (potentially nested) synchronized products of basic components. Thus, we first show that wellstructure is preserved under synchronized product. Our presentation of well-structured transition systems differs from (and generalizes) the standard (non-labeled) one as we need to take care of labels.

Until the end of this sub-section, we assume that each set of labels $\Sigma$ is partitioned into a set $\Sigma_{\tau}$ of local labels (for internal transitions) and a set $\Sigma_{\gamma}$ of global labels (for synchronizable transitions). In order to account for this separation between internal transitions and synchronizable ones, we assume (1) that every synchronization constraint $V$ implicitly contains the set $V_{\tau}=\{\langle\tau, \varepsilon, \ldots, \varepsilon\rangle, \ldots,\langle\varepsilon, \ldots, \varepsilon, \tau, \varepsilon, \ldots, \varepsilon\rangle, \ldots$, $\left.\langle\varepsilon, \ldots, \varepsilon, \tau\rangle / \tau \in \Sigma_{\tau}\right\}$ of synchronization vectors, and (2) that no local label $\tau \in \Sigma_{\tau}$ may appear in a synchronization vector of $V \backslash V_{\tau}$. Naturally $V_{\tau}$ becomes the set of local labels of any synchronized product w.r.t. $V$.

A preordered LTS is any LTS $\mathcal{S}=\left(S, s^{0}, \Sigma, \rightarrow\right)$ equipped with a preorder $\preceq$ on $S$. We say that $\preceq$ is compatible (resp. transitively compatible, reflexively compatible) with 
$\rightarrow$ if for every transition $s \stackrel{a}{\rightarrow} s^{\prime}$ and $t \succeq s$ there exists $t^{\prime} \succeq s^{\prime}$ such that $t \stackrel{\sigma}{\rightarrow} t^{\prime}$ for some $\sigma \in \Sigma^{*}$ satisfying:

$$
\begin{aligned}
& \left\{\begin{array} { l l } 
{ \sigma \in \Sigma _ { \tau } ^ { * } } & { \text { if } a \in \Sigma _ { \tau } } \\
{ \sigma \in \Sigma _ { \tau } ^ { * } a \Sigma _ { \tau } ^ { * } \text { otherwise } }
\end{array} \quad \left\{\begin{array} { l } 
{ \sigma \in \Sigma _ { \tau } ^ { + } \quad \text { if } a \in \Sigma _ { \tau } } \\
{ \sigma \in \Sigma _ { \tau } ^ { * } a \Sigma _ { \tau } ^ { * } \text { otherwise } }
\end{array} \quad \left\{\begin{array}{l}
\sigma \in\{\varepsilon\} \cup \Sigma_{\tau} \text { if } a \in \Sigma_{\tau} \\
\sigma=a
\end{array}\right.\right.\right. \\
& \text { (compatibility) (transitive compatibility) (reflexive compatibility) }
\end{aligned}
$$

Moreover we also say that $\preceq$ is strictly compatible with $\rightarrow$ if both $\preceq$ and $\prec$ are compatible with $\rightarrow$ (recall that $s \prec s^{\prime}$ is defined by $s \preceq s^{\prime} \npreceq s$ ). Of course, this strictness notion may be combined with transitive and reflexive compatibilities.

Remark 3.1. The previous definitions of compatibility coincide with the definitions given in [FS01] when $\Sigma=\Sigma_{\tau}$ is a singleton.

Any synchronized product $\mathcal{S}_{\otimes}$ of $n$ preordered LTSs $\left(\mathcal{S}_{1}, \preceq_{1}\right), \ldots,\left(\mathcal{S}_{n}, \preceq_{n}\right)$ may be equipped with the product preorder $\preceq_{\otimes}$ defined by $\boldsymbol{s} \preceq_{\otimes} \boldsymbol{s}^{\prime}$ iff $\boldsymbol{s}(i) \preceq_{i} \boldsymbol{s}^{\prime}(i)$ for every $1 \leq i \leq n$. The following proposition shows that all six compatibility notions defined above are preserved under synchronized product.

Proposition 3.2. Let Cond denote any compatibility condition among $\{($ non-strict $)$, strict $\} \times\{($ standard $)$, transitive, reflexive $\}$. Any synchronized product of preordered LTSs with compatibility Cond also has compatibility Cond.

Recall that a well-preorder on some set $U$ is any preorder $\preceq$ on $U$ such that any infinite sequence $x_{1}, \ldots, x_{k}, \ldots$ of elements in $U$ contains an increasing pair $x_{i} \preceq x_{j}$ with $i<j$. A well-preordered LTS is any preordered LTS $(\mathcal{S}, \preceq)$ where $\preceq$ is a well-preorder on the state set $S$ of $\mathcal{S}$. Since the product preorder of any $n$ well-preorders is also a wellpreorder (from Higman's lemma), we obtain that well-preordering is preserved under synchronized product.

Proposition 3.3. Any synchronized product of well-preordered LTSs is a well-preordered LTS.

A well-structured LTS is any well-preordered LTS with (standard) compatibility. It follows from the two previous propositions that well-structure is preserved under synchronized product.

\subsection{Finite Property-Preserving Truncation of Well-Structured LES}

The intuition behind well-structure is that any state may be weakly simulated by any greater state, and thus we may forget about smaller states when performing reachability analysis. The well-preordering condition between states guarantees termination of the analysis [FS01]. We show in this sub-section how to extend these ideas to the partialorder verification of well-structured labeled transition systems.

Recall that any marked LES $(\mathcal{E}, M)$ induces a labeled transition system $\mathcal{S}_{\mathcal{E}}^{M}$. We lift the well-structure notions defined in the previous sub-section from labeled transition systems to labeled event structures. A preordered marked LES (resp. well-preordered marked LES) is any marked LES $(\mathcal{E}, M)$ equipped with a preorder (resp. well-preorder) 
$\preceq$ on $M\left[\mathcal{C}_{f}(\mathcal{E})\right]$. Given any preordered marked LES $(\mathcal{E}, M, \preceq)$, we say that $(\mathcal{E}, M, \preceq)$ has compatibility Cond $\in\{$ (non-strict), strict $\} \times\{$ (standard), transitive, reflexive $\}$ whenever $\mathcal{S}_{\mathcal{E}}^{M}$ has compatibility Cond.

Consider any preordered marked LES $(\mathcal{E}, M, \preceq)$ where $\mathcal{E}=(E, \leq, \#, \Sigma, l)$. A cutoff event is any $e_{\text {cut }} \in E$ such that $M\left(\left[e_{c u t}\right]\right) \succeq M([e])$ for some event $e$ with $e<e_{\text {cut }}$. The truncation $\mathcal{T}(\mathcal{E}, M, \preceq)$ of $(\mathcal{E}, M, \preceq)$ is the set of events having no strictly causal cutoff event, formally $\mathcal{T}(\mathcal{E}, M, \preceq)=E \backslash\left\{e \in E / \exists e_{\text {cut }} \in E_{\text {cut }}, e_{\text {cut }}<e\right\}$ where $E_{\text {cut }}$ denotes the set of cutoff events in $\mathcal{E}$. Observe that $\mathcal{T}(\mathcal{E}, M, \preceq)$ is downward-closed, and that any minimal cutoff event (i.e. any event in $\operatorname{Min}_{\leq}\left(E_{c u t}\right)$ ) is a maximal event of $\mathcal{T}(\mathcal{E}, M, \preceq)$ but the converse does not hold in general. In order to preserve termination and boundedness properties, this truncation criterion "respects" causality, and this leads to larger truncations than in [McM95] where the truncation only preserves reachability properties.

We will show in the rest of this sub-section how to use the truncation to decide several verification problems. Unfortunately the truncation may be infinite in general, as it may be "too deep" and/or "too wide". A well-preordering condition avoids the first possibility, and a branching finiteness assumption eliminates the second.

Proposition 3.4. The truncation of any well-preordered finitely-branching marked LES is finite.

Given any labeled transition system $\mathcal{S}$, we say that $\mathcal{S}$ terminates (resp. is bounded) if $\mathcal{S}$ has no infinite execution (resp. has a finite reachability set post*). The two following propositions show that, assuming an adequate compatibility condition, the truncation defined above contains enough information to decide termination and boundedness. Remark that in these two propositions, the finiteness requirement on the truncation can be dropped when the marked LES is finitely-branching and well-preordered.

Proposition 3.5. For any preordered finitely-branching marked LES $(\mathcal{E}, M, \preceq)$ with transitive compatibility, $\mathcal{S}_{\mathcal{E}}^{M}$ terminates iff $\mathcal{T}(\mathcal{E}, M, \preceq)$ is finite and contains no cutoff event.

In order to decide boundedness, we will need "strict" cutoff events, and we will also require a partial-order $\preceq$. Formally, a strict cutoff event is any $e_{c u t} \in E$ such that $M\left(\left[e_{c u t}\right]\right) \succ M([e])$ for some event $e$ with $e<e_{\text {cut }}$. Observe that any strict cutoff event is also a cutoff event. A partially-ordered marked LES is any preordered marked LES $(\mathcal{E}, M, \preceq)$ where $\preceq$ is a partial order on $M\left[\mathcal{C}_{f}(\mathcal{E})\right]$. Notice that the following proposition does not hold for general preordered marked LES.

Proposition 3.6. For any partially-ordered marked LES $(\mathcal{E}, M, \preceq)$ with strict compatibility, $\mathcal{S}_{\mathcal{E}}^{M}$ is bounded iff $M\left[\left\{C \in \mathcal{C}_{f}(\mathcal{E}) / C \subseteq \mathcal{T}(\mathcal{E}, M, \preceq)\right\}\right]$ is finite and $\mathcal{T}(\mathcal{E}, M, \preceq)$ contains no strict cutoff event.

We now turn our attention to the quasi-liveness problem which, assuming an adequate compatibility condition, reduces to the computation of the upward closure of post ${ }_{\delta_{\varepsilon}^{M}}^{*}$. For any labeled transition system $\mathcal{S}=\left(S, s^{0}, \Sigma, \rightarrow\right)$, we say that a given label $a \in$ $\Sigma$ is quasi-live if there is an execution in $\mathcal{S}$ containing a transition labeled with $a$. 
The truncation that we have used so far would be sufficient to decide quasi-liveness, but in order to improve efficiency, we consider a refined notion of cutoff events which leads to smaller truncations (that still contain enough information to decide quasi-liveness). This refined notion is based on the size of configurations as in [McM95]. Formally, given any preordered marked LES $(\mathcal{E}, M, \preceq)$ where $\mathcal{E}=(E, \leq, \#, \Sigma, l)$, we denote by $\unlhd$ the preorder on $\mathcal{C}_{f}(\mathcal{E})$ defined by $C \unlhd C^{\prime}$ iff $\operatorname{Card}(C) \leq \operatorname{Card}\left(C^{\prime}\right)$. Note that $C \triangleleft C^{\prime}$ means $\operatorname{Card}(C)<\operatorname{Card}\left(C^{\prime}\right)$. A $\triangleleft$-cutoff event is any $e_{\text {cut }} \in E$ such that $M\left(\left[e_{c u t}\right]\right) \succeq M([e])$ for some event $e$ with $[e] \triangleleft\left[e_{\text {cut }}\right]$. The $\triangleleft$-truncation $\mathcal{T}_{\triangleleft}(\mathcal{E}, M, \preceq)$ of $(\mathcal{E}, M, \preceq)$ is the set of events having no strictly causal $\triangleleft$-cutoff event, formally $\mathcal{T}_{\triangleleft}(\mathcal{E}, M, \preceq)=E \backslash\left\{e \in E / \exists e_{c u t} \in E_{c u t}^{\triangleleft}, e_{c u t}<e\right\}$ where $E_{c u t}^{\triangleleft}$ denotes the set of $\triangleleft$-cutoff events in $\mathcal{E}$.

For clarity, any (standard) cutoff event will now be called a $\subset$-cutoff event, and the (standard) truncation will now be called the $\subset$-truncation and be denoted by $\mathcal{T}_{\subset}(\mathcal{E}, M$, $\preceq)$. It is readily seen that $\mathcal{T}_{\triangleleft}(\mathcal{E}, M, \preceq) \subseteq \mathcal{T}_{\subset}(\mathcal{E}, M, \preceq)$. Hence $\triangleleft$-truncations are also finite for well-preordered finitely-branching marked LESs. Notice that the following proposition requires reflexive compatibility of the inverse preorder $\succeq$ of $\preceq$ (this requirement was called "downward compatibility" in [FS01]).

Proposition 3.7. For any preordered marked LES $(\mathcal{E}, M, \succeq)$ with reflexive compatibility, the two following assertions hold:

i) the sets $M\left[\left\{C \in \mathcal{C}_{f}(\mathcal{E}) / C \subseteq \mathcal{T}_{\triangleleft}(\mathcal{E}, M, \preceq)\right\}\right]$ and post ${ }_{\mathcal{S}_{\mathcal{E}}^{M}}^{*}$ have the same upward closure w.r.t. $\preceq$.

ii) for any global label $a$, a is quasi-live in $\mathcal{S}_{\mathcal{E}}^{M}$ iff a labels an event in $\mathcal{T}_{\triangleleft}(\mathcal{E}, M, \preceq)$.

Remark that the previous proposition also holds for the standard truncation (i.e. we may replace $\mathcal{T}_{\triangleleft}$ by $\mathcal{T}_{\subset}$ in the proposition). We may even further refine the truncation by considering a preorder on $\mathcal{C}_{f}(\mathcal{E})$ that refines $\unlhd$ (i.e. a preorder that is contained in $\unlhd$ ). However Proposition 3.7 may not hold for this refined preorder unless we assume stronger requirements on the preordered marked LES $(\mathcal{E}, M, \succeq)$. In particular, if every label is global then Proposition 3.7 still holds for the lexicographic preorder between configurations defined in [ERV02].

\section{Compositional Unfoldings of Concurrent Systems}

We now give algorithms for unfolding given systems into labeled event structures. Figure 4(a) depicts an LES $\varepsilon_{a}$ modeling a positive counter initialized to 1. Black (resp. white) events represent increasing $(+)$ events (resp. decreasing (-) events) and arrows represent the causality relation. Since this counter is initialized to 1 , both - and + are initially enabled, however one needs to first unfold a + event before unfolding a second -, and so on. Thus, unfolding $\mathcal{E}_{a}$ is achieved by first building the lowest two events (initialization phase), and then extending every + event with new - and + events (extension phase).

All our unfolding algorithms rely on this principle. The following Unfold builds onthe-fly LES for given systems: 


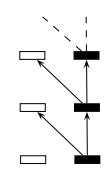

(a)

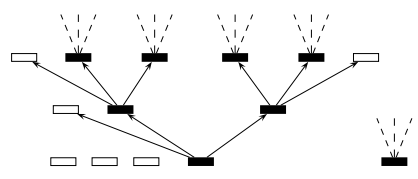

(b)

Fig. 1. LES for counters with: (a) $v_{0}=1$ and $k=1$, (b) $v_{0}=3$ and $k=2$

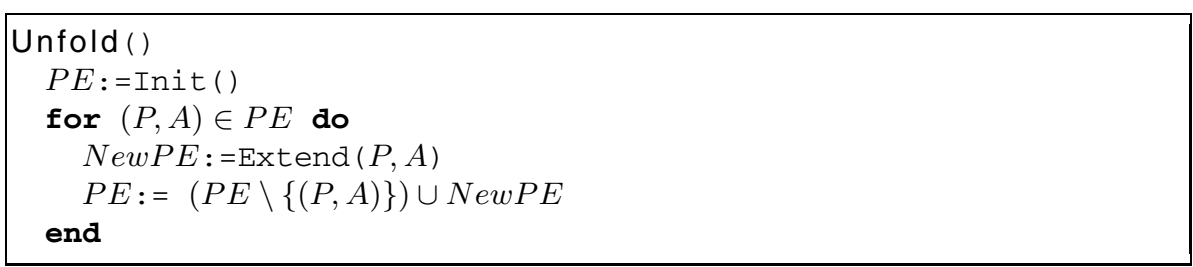

Pairs $(P, A)$ correspond to new extensions: $P$ is the preset of the new event (e.g. the lowest black event in Figure $4(\mathrm{a}))$ and $A$ is the set of actions to extend with (e.g. $\{+,-\})$. Extending creates new events using the NewEvent function that also updates causality and conflict relations. Then Unfold computes new pending extensions. Notice that this algorithm terminates if Extend eventually always returns an empty set, which is the case for well-structured LESs if we do not extend cut-off events as defined in section 3.2 .

In the sequel, we detail Init and Extend functions for three types of systems. We first consider counters for which we give dedicated functions. Ad hoc algorithms are always more efficient and can be defined for other datatypes for instance FIFO queues [LI05]. However, it is not always possible nor wanted to have specific algorithms, hence in section 4.2 we define functions that compute a concurrent marked LES for any given LTS. Finally, in section 4.3 , we consider the unfolding of synchronized products of systems.

\subsection{Unfolding Counters}

A counter is a datatype with values ranging over the set of natural numbers $\mathbb{N}$, equipped with two operations: + and - that respectively increase and decrease its value, and initial value $v_{0} \in \mathbb{N}$. It may be viewed as an LTS $\mathcal{S}_{c}=\left(\mathbb{N}, v_{0},\{+,-\}, \rightarrow\right)$ where $n \stackrel{+}{\rightarrow} n+1$ for any $n \in \mathbb{N}$ and $n \stackrel{-}{\rightarrow} n-1$ for all $n>0$. Places of Petri nets are examples of such counters.

We aim at defining Init and Extend functions that build an LES for a counter. Figure 4 depicts two different LESs $\mathcal{E}_{a}$ and $\mathcal{E}_{b}$ modeling a counter. The labeling $l_{c}$ associates + (resp. - ) to every black (resp. white) event and the natural marking $M_{c}$ associates to every $C \in \mathcal{C}_{f}(\mathcal{E})$ the value $v_{0}+\operatorname{Card}\left(\left\{e \in C / l_{c}(e)=+\right\}\right)-\operatorname{Card}(\{e \in$ $\left.\left.C / l_{c}(e)=-\right\}\right)$. Both $\left(\varepsilon_{a}, M_{c}\right)$ and $\left(\mathcal{E}_{b}, M_{c}\right)$ are marked LESs for $\mathcal{S}_{c}$.

In these LESs, causality between - and + events correspond to intuitive constraints: a counter must be increased before being decreased. However, if $v_{0}>0$, it may be decreased $v_{0}$ times without any increasing. Also, + events are concurrent since there is no constraint for increasing. Hence, labeled event structures $\mathcal{E}_{a}$ and $\mathcal{E}_{b}$ differ in the degree 
of concurrency between + events. Choosing the degree $k \geq 1$ of concurrency is a matter of modeling leading to more or less concurrent truncations depending on the system that is analysed, in particular for synchronized products of LESs (see section 4.3).

Init creates $v_{0}(\emptyset,\{-\})$ and $k(\emptyset,\{+\})$ pending extensions. Then, Extend simply follows the the principle depicted in Figures 4 (a) and 4 b).

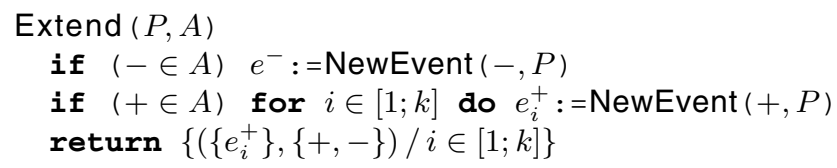

Using our algorithm, one obtains the $\left(v_{0}=1, k=1\right)$ counter LES in Figure 4(a), which corresponds to McMillan's unfolding of a counter [McM95]. However, Figure 4(b) shows that our approach yields the ability to choose more or less concurrent models using parameter $k$.

\subsection{Unfolding Labeled Transition Systems}

Defining the semantics of given systems as LESs or designing dedicated unfolding algorithms for those systems is often very hard. However, most systems can easily be described as LTSs. Hence, being able to compute a marked LES for any LTS is a solution to benefit from intrinsic concurrency in those systems.

A trivial LES for any LTS is its reachability tree, however every event in a reachability tree is either in causality or in conflict with any other event. We introduce an algorithm that computes a concurrent marked LES for any given LTS. Figure 2(b) depicts a prefix of the LES $\varepsilon_{f}$ computed by our algorithm for a FIFO queue LTS $\delta_{f}$ over messages $\{a, b\}$. Concurrency essentially corresponds to independence diamonds in $\mathcal{S}_{f}$ : whenever two or more actions are commutative. Moreover, our algorithm infers local concurrency: the same actions can be concurrent in some state of $\mathcal{S}_{f}$ and conflicting in some other state.

Init defines initially pending extension $(\emptyset, \Sigma)$ and marking $M(\emptyset)=s^{0}$ for the given LTS $\left(S, s^{0}, \Sigma, \rightarrow\right)$. Assume that $e_{0}$ in Figure 2 b) has not been extended so far: $P=$ $\left\{e_{0}\right\}$ and $A=\{? a, ? b, ! a, ! b\}$. Extending $P$ results in creating new events $\left\{e_{2}, e_{3}, e_{4}\right\}$ in causality with $e_{0}\left(? b\right.$ is not enabled in $\left.M\left(\left\{e_{0}\right\}\right)\right)$. Now, extending $P=\left\{e_{0}, e_{2}\right\}$ with label ! $a$ does not create any event since adding $e_{3}$ to $P$ yields the expected extension. Hence, our Extend function first looks for concurrent events that can extend $P$, and

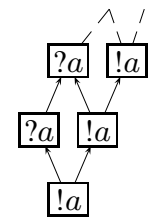

(a)

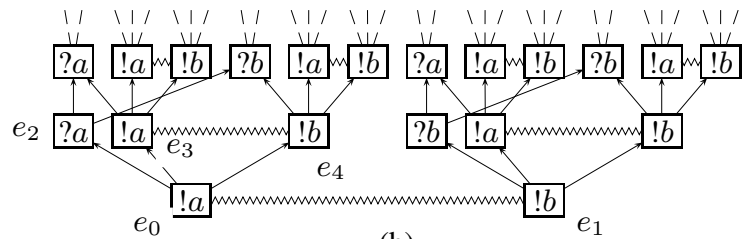

(b)

Fig. 2. LES for $\emptyset$-initialized FIFO channels with messages: (a) $\{a\}$ and (b) $\{a, b\}$ 
then creates new events only for the labels in $A \backslash l(X)$ that were not matched by this first step.

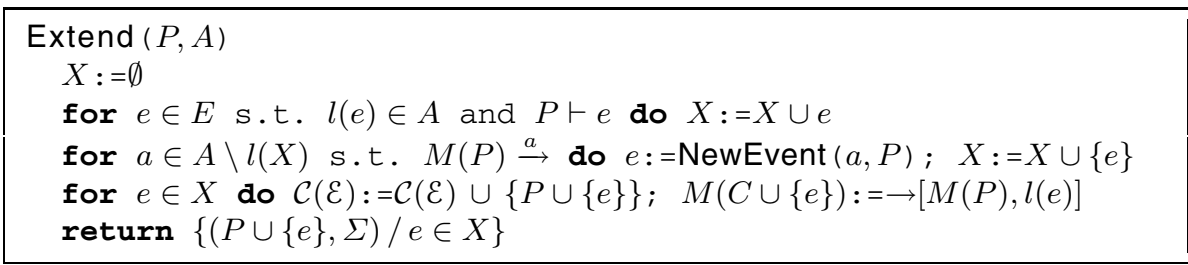

Notice that in this algorithm, $P$ is always a configuration: Extend explores the configuration space of the LES.

However, Extend is not correct so far as it does not add any conflict whereas Figure 2 b) clearly shows the need for it. Missing conflicts are detected as follows. Assume that $\mathcal{E}_{f}$ in Figure 2(b) only contains $e_{0}$ and $e_{1}$ without conflict so far. Extending $\left(\left\{e_{0}\right\}, ! b\right)$ leads to configuration $\left\{e_{0}, e_{1}\right\}$ with $M\left(\left\{e_{0}, e_{1}\right\}\right)=\rightarrow\left[M\left(\left\{e_{0}\right\}\right), ! b\right]=a b$. Next, extending $\left(\left\{e_{1}\right\}, ! a\right)$, leads to associating $e_{0}$ to $\left\{e_{1}\right\}$ which results to be impossible since $\rightarrow\left[M\left(\left\{e_{1}\right\}\right), ! a\right]=b a \neq M\left(\left\{e_{0}, e_{1}\right\}\right)$. Hence, conflict must be added between $e_{0}$ and $e_{1}$ using the CheckConflict function below when Extend detects the problem.

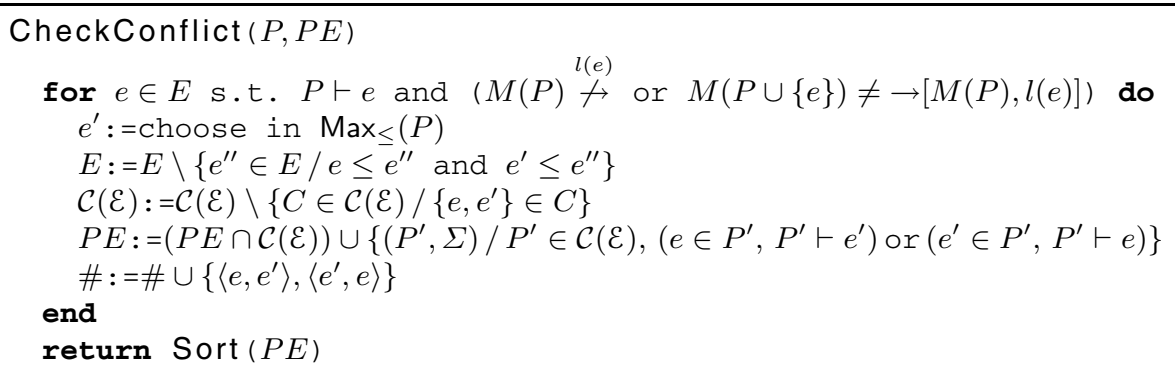

CheckConflict updates $P E$ since whenever one needs to add conflict between 2 events $e_{0}$ and $e_{1}$, every configuration in $\mathcal{C}(\mathcal{E})$ that contains both events must be discarded and every configuration that contains $e_{0}$ (resp. $e_{1}$ ) has potentially mistaken extensions. Notice that pending extensions $(P, A)$ in $P E$ are eventually sorted w.r.t increasing size of $P$. This is due to a natural hypothesis made by Extend: if $P$ is to be extended, then all the extensions of any $P^{\prime} \subset P$ are up-to-date.

Figure 2 depicts the marked LES obtained for LTS modeling FIFO queues in the standard way (one state per queue content, and transitions w.r.t. FIFO policy) by applying our algorithm. They exactly correspond to the LES computed by the method in [LI05].

\subsection{Unfolding Synchronized Products of Components}

Sections 4.1 and 4.2 present unfolding algorithms for single components. We now introduce an algorithm for unfolding complex systems built from synchronized components. 


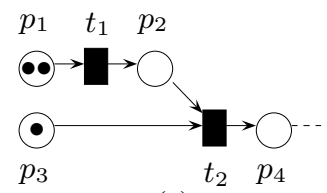

(a)

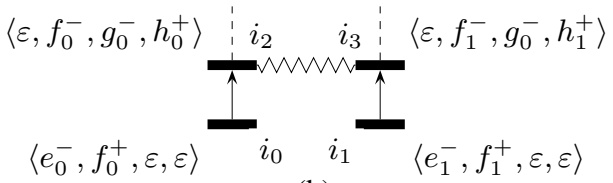

(b)

Fig. 3. A Petri net $N$ (a) and a marked LES for $N$ (b)

Consider Petri net $N$ in Figure 3(a). In our framework, each place $p_{i}$ is modeled by a counter LES and each transition $t_{j}$ by a synchronization vector between actions of these counters. Since tokens in Petri nets are concurrent processes, we choose a $\left(v_{0}=2, k=\right.$ 2 ) counter LES $\mathcal{E}_{1}$ for $p_{1}$ since it initially contains 2 tokens. Similarly we choose a $(1,1)$ counter LES $\mathcal{E}_{3}$ for $p_{3}$. Place $p_{2}$ is initially empty and can simultaneously contain 2 tokens, thus we model it by a $(0,2)$ counter LES $\varepsilon_{2}$. Finally, we choose a $(0,1)$ counter LES $\mathcal{E}_{4}$ for $p_{4}$. In the case of unbounded places, one can choose $k$ as the number of entering edges.

Let $e_{i}^{a}$ (resp. $f_{i}^{a}, g_{i}^{a}$ and $h_{i}^{a}$ ) denote the ith event labeled by $a \in\{+,-\}$ in $\mathcal{E}_{1}$ (resp. $\varepsilon_{2}, \varepsilon_{3}$ and $\varepsilon_{4}$ ) w.r.t. causality. The semantics of $N$ is modeled in the synchronized product of $\mathcal{E}_{1}, \mathcal{E}_{2}, \mathcal{E}_{3}$ and $\mathcal{E}_{4}$ by the synchronization vectors $\langle-,+, \varepsilon, \varepsilon\rangle$ for $t_{1}$ and $\langle\varepsilon,-,-,+\rangle$ for $t_{2}$.

Figure 3 (b) depicts LES $\varepsilon_{N}$ obtained for $N$ using our unfolding algorithm. To each event in $\varepsilon_{N}$ is associated a tuple of components' events by mapping $\lambda:\left(E_{1} \cup\{\varepsilon\}\right) \times$ $\cdots \times\left(E_{n} \cup\{\varepsilon\}\right) \rightarrow E$, for instance $\lambda\left(i_{0}\right)=\left\langle e_{0}^{-}, f_{0}^{+}, \varepsilon, \varepsilon\right\rangle$. Conflict and causality relations in $\mathcal{E}_{N}$ are defined from components' ones. Basically, conflict appears when a components' event is used by two or more global events, e.g. $g_{0}^{-}$in $i_{2}$ and $i_{3}$, and causality inherits from components, e.g. $f_{0}^{+} \rightarrow f_{0}^{-}$entails $i_{0} \rightarrow i_{2}$. Formally, let $\left\langle e_{1}, \ldots, e_{n}\right\rangle \#\left\langle e_{1}^{\prime}, \ldots, e_{n}^{\prime}\right\rangle$ iff there exists $i$ s.t. $e_{i}=e_{i}^{\prime}$ or $e_{i} \#_{i} e_{i}^{\prime}$. The global causality and conflict relations are respectively the smallest partial order $\leq$ and the smallest symmetric and irreflexive relation \# satisfying for every global events $e, e^{\prime}, e^{\prime \prime}$ :

- if $e \# e^{\prime}$ and $e^{\prime} \leq e^{\prime \prime}$ then $e \# e^{\prime \prime}$, and

- if $\lambda(e) \# \lambda\left(e^{\prime}\right)$ then $e \# e^{\prime}$, and

- if there exists $i$ s.t. $(\lambda(e))_{i} \leq_{i}\left(\lambda\left(e^{\prime}\right)\right)_{i}$ and we do not have $e \# e^{\prime}$ then $e \leq e^{\prime}$.

As Figure 3 (b) shows, unfolding a synchronized product of LESs consists in associating components' events into global events w.r.t. synchronization vectors, conflict and causality relations. Since components' LES maybe infinite we use an on-the-fly algorithm that proceeds as follows. Init initializes every component (in particular $P E_{i}$ ) and extends all their initially pending extensions $\left(\emptyset, A_{i}\right)$. This is necessary due to synchronization. Next, extending $(P, A)$ in the global LES consists, for every synchronization vector $v \in A$, in finding all tuples $\left\langle e_{1}, \ldots, e_{n}\right\rangle$ of components' events which are instances of $v$ that extend $P$. A new global event $e$ is created for each such instance $\left\langle e_{1}, \ldots, e_{n}\right\rangle$ and each conflict-free preset $p s$ of global events that match the presets of every $e_{i}$. Finally, every component such that $e_{i} \neq \varepsilon$ is extended since the successors of $e_{i}$ may be needed to extend further. 


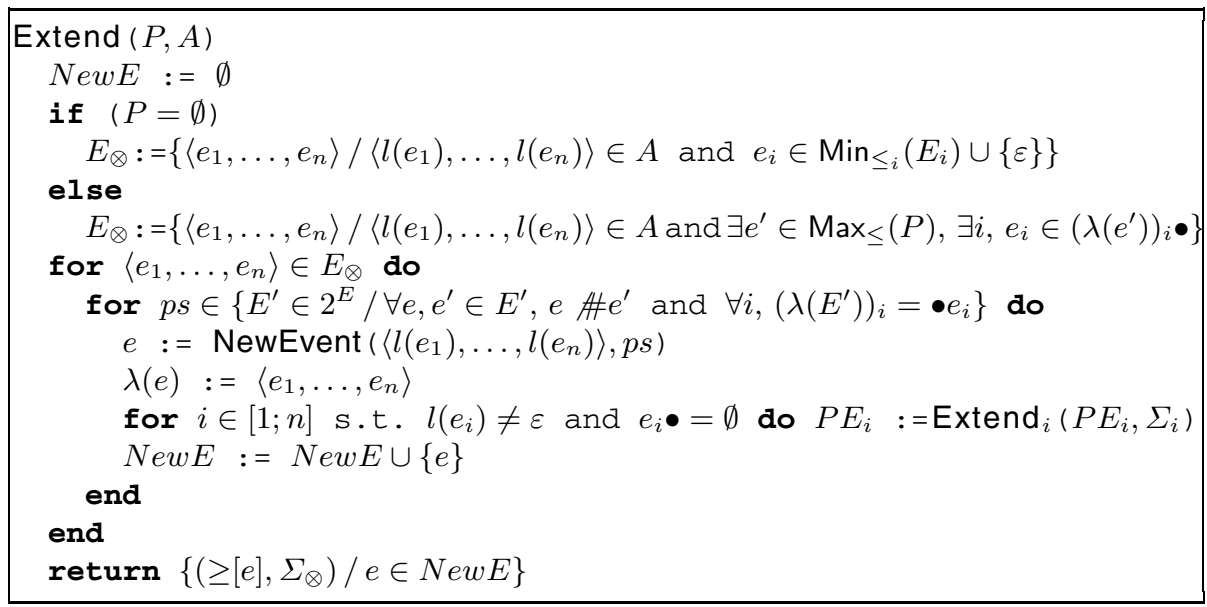

In this algorithm, we denote by $\bullet e=\operatorname{Max}_{\leq}((\geq[e]) \backslash\{e\})$ the preset of $e$ w.r.t. causality, and by $e \bullet=\operatorname{Min}_{\leq}((\leq[e]) \backslash\{e\})$ the postset of $e . \Sigma_{i}$ denotes the set of actions of component $i$. Notice that $\operatorname{Extend}_{i}\left(P E_{i}, \Sigma_{i}\right)$ is a slight abuse of notations as $P E_{i}$ is a set of pending extensions.

Extend first checks that components' events have not been extended yet before doing so $\left(e_{i} \bullet=\emptyset\right)$ since an event may be associated to many global events. The labeling of global events and configurations are defined component-wise, and global conflict and causality relations are computed as defined previously.

Using our algorithm, one can compute a marked unfolding $\mathcal{E}_{\otimes}$ of a synchronized product of components as depicted in Figure 3 Furthermore, $\mathcal{E}_{\otimes}$ can itself be used as a component, giving raise to hierarchical unfolding of systems and components.

\section{Experimental Results}

We have implemented the algorithms and truncation techniques presented in this paper in a tool called ESU. This tool is implemented in Objective Caml, and permits the verification of termination, boundedness and quasi-liveness for synchronized products of well-structured components. Components may be counters, queues or finite-state (control) automata. For the particular case of bounded systems such as bounded Petri nets, ESU is also able to compute reachability set.

To our knowledge, ESU is the first tool able to analyse infinite-state systems using forward unfolding techniques. Hence, in order to evaluate the benefits of our approach we have compared ESU with two tools for Petri nets: the PEP environment which provides an unfolding tool for bounded Petri nets [Pep], and the tool TINA which analyzes arbitrary Petri nets using structural analysis techniques and forward Karp-Miller reachability analysis [Tin]. Petri nets are modeled in ESU by synchronized counter components. Experiments were conducted on an Intel XEON 2.2 GHz station with $6 \mathrm{~GB}$ of RAM. In the following tables, $E$ (resp. $E_{c f}, N, S$ ) denotes the number of events in the truncation (resp. of cutoff events, of nodes in TINA's tree, of markings in TINA's tree), and a '-' means that the analysis exhausted memory or did not finish within 10 minutes. 
The Petri net depicted below represents a concurrent Producer/Consumer Petri Net with $n$ independent production lines and $m$ machines on each line. The products from these $n$ lines are combined into another product that is then stored in place $p_{s}$. PEP's unfolder cannot analyze this Petri net as it is unbounded. EsU performs very well on this example, but this is not very surprising as this Petri net is extremely concurrent. Observe that the number of events in the truncation is approximately the number of transitions in the Petri net.

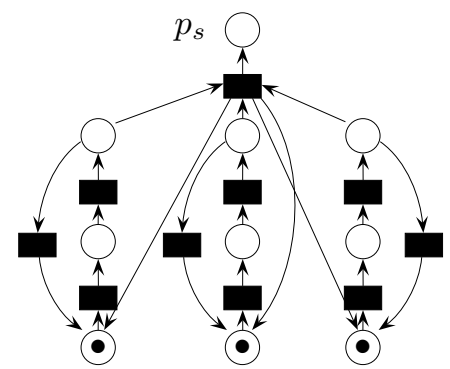

\begin{tabular}{|l||r|r||r|r|r|}
\hline \multicolumn{1}{|c||}{} & \multicolumn{2}{c||}{ TINA } & \multicolumn{3}{c|}{ ESU } \\
\hline$m \times n$ & $N$ & $\mathrm{~T}(\mathrm{~s})$ & $E$ & $E_{c f}$ & $\mathrm{~T}(\mathrm{~s})$ \\
\hline \hline $3 \times 3$ & 49 & 0.01 & 10 & 4 & 0.01 \\
\hline $5 \times 5$ & 4636 & 0.04 & 25 & 5 & 0.01 \\
\hline $7 \times 7$ & 1094241 & 24.41 & 50 & 8 & 0.01 \\
\hline $7 \times 10$ & - & - & 71 & 8 & 0.03 \\
\hline $10 \times 10$ & - & - & 96 & 6 & 0.04 \\
\hline $20 \times 25$ & - & - & 491 & 11 & 1.4 \\
\hline
\end{tabular}

We also experimented on a more challenging and well-known example: the swimming pool. The swimming pool has much less explicit concurrency as most transitions share places. We used TINA's bounded swimming pool Petri net which is a variant of the classical one with an additional place that limits the number of clients [Tin]. In the following table, the size denotes the number of resources in the swimming pool.

\begin{tabular}{|r||r|r|r||r|r|r||r|r|r|}
\hline \multicolumn{1}{|r||}{} & \multicolumn{3}{c||}{ PEP } & \multicolumn{3}{c||}{ TINA } & \multicolumn{3}{|c|}{ ESU } \\
\hline Size & $E$ & $E_{c f}$ & $\mathrm{~T}(\mathrm{~s})$ & $N$ & $S$ & $\mathrm{~T}(\mathrm{~s})$ & $E$ & $E_{c f}$ & $\mathrm{~T}(\mathrm{~s})$ \\
\hline \hline 3 & 37593 & 18009 & 159.59 & 126 & 56 & 0.00 & 18 & 3 & 0.01 \\
\hline 10 & - & - & - & 12012 & 3003 & 0.05 & 60 & 10 & 0.20 \\
\hline 20 & - & - & - & 255024 & 53130 & 3.35 & 120 & 20 & 3.02 \\
\hline 30 & - & - & - & 1669536 & 324632 & 44.74 & 180 & 30 & 20.64 \\
\hline 40 & - & - & - & 6516048 & 1221759 & 297.19 & 240 & 40 & 64.04 \\
\hline
\end{tabular}

Future work will focus on improving and extending our method to other frameworks for the analysis of infinite state systems. In particular we plan to focus on abstraction algorithms in order to build more compact and concurrent event structures that would abstract away causality and conflict information that is irrelevant w.r.t. to a desired property. We also plan to consider acceleration techniques as a tool for truncating unfoldings, hence enforcing the termination of our algorithms while preserving reachability properties.

Acknowledgements. The authors wish to thank Igor Walukiewicz for insightful comments and suggestions on a preliminary version of this paper.

\section{References}

[AČJT00] P. A. Abdulla, K. Čerāns, B. Jonsson, and Y. K. Tsay. Algorithmic analysis of programs with well quasi-ordered domains. Information and Computation, 160(12):109-127, 2000. 
[AIN00] P. A. Abdulla, S. P. Iyer, and A. Nylén. Unfoldings of unbounded petri nets. In Proc. of 12th Int. Conf. on Computer Aided Verification (CAV'00), volume 1855 of Lecture Notes in Computer Science, pages 495-507. Springer, 2000.

[AJKP98] P. A. Abdulla, B. Jonsson, M. Kindahl, and D. Peled. A general approach to partial order reductions in symbolic verification (extended abstract). In Proc. of 10th Int. Conf. on Computer Aided Verification (CAV '98), volume 1427 of Lecture Notes in Computer Science, pages 379-390. Springer, 1998.

[Arn94] A. Arnold. Finite Transition Systems. Semantics of Communicating Systems. Prentice Hall Int., 1994.

[CGP01] J-M. Couvreur, S. Grivet, and D. Poitrenaud. Unfolding of products of symmetrical petri nets. In Proc. 22nd Int. Conf. on Application and Theory of Petri Nets (ICATPN'01), volume 2075 of Lecture Notes in Computer Science, pages 121-143. Springer, 2001.

[DJN04] J. Desel, G. Juhás, and C. Neumair. Finite unfoldings of unbounded petri nets. In Proc. 25th Int. Conf. on Applications and Theory of Petri Nets (ICATPN'04), volume 3099 of Lecture Notes in Computer Science, pages 157-176. Springer, 2004.

[ER99] J. Esparza and S. Römer. An unfolding algorithm for synchronous products of transition systems. In 10th Int. Conf. on Concurrency Theory (CONCUR'99), volume 1664 of Lecture Notes in Computer Science, pages 2-20. Springer, 1999.

[ERV02] J. Esparza, S. Römer, and W. Vogler. An improvement of McMillan's unfolding algorithm. Formal Methods in System Design, 20(3):285-310, 2002.

[Fin90] A. Finkel. Reduction and covering of infinite reachability trees. Information and Computation, 89(2):144-179, 1990.

[FS01] A. Finkel and P. Schnoebelen. Well-structured transition systems everywhere! Theoretical Computer Science, 256(1-2):63-92, 2001.

[God96] P. Godefroid. Partial-order methods for the verification of concurrent systems: An approach to the state-explosion problem, volume 1032 of Lecture Notes in Computer Science. Springer, New York, NY, USA, 1996.

[KK03] V. Khomenko and M. Koutny. Branching processes of high-level petri nets. In Proc. 9th Int. Conf. on Tools and Algorithms for the Construction and Analysis of Systems (TACAS'03), volume 2619 of Lecture Notes in Computer Science, pages 458-472. Springer, 2003.

[LI05] Y. Lei and S. P. Iyer. An approach to unfolding asynchronous communication protocols. In Proc. 13th Int. Symp. on Formal Methods (FM'05), volume 3582 of Lecture Notes in Computer Science, pages 334-349. Springer, 2005.

[Maz86] A. W. Mazurkiewicz. Trace theory. In Advances in Petri Nets, volume 255 of Lecture Notes in Computer Science, pages 279-324. Springer, 1986.

[McM95] K. L. McMillan. A technique of state space search based on unfolding. Formal Methods in System Design, 6(1):45-45, 1995.

[NPW81] M. Nielsen, G. Plotkin, and G. Winskel. Petri nets, event structures and domains, part I. Theoretical Computer Science, 13:85-108, 1981.

[NRT95] M. Nielsen, G. Rozenberg, and P. S. Thiagarajan. Transition systems, event structures and unfoldings. Information and Computation, 118(2):191-207, 1995.

[Pe193] D. Peled. All from one, one for all: on model checking using representatives. In Proc. of the 5th Int. Conf. on Computer Aided Verification (CAV'93), volume 697 of Lecture Notes in Computer Science, pages 409-423. Springer, 1993.

[Pep] PEP tool. Homepage: http: //peptool.sourceforge.net/.

[Tin] TINA tool. Homepage: http://www.laas.fr/tina/.

[Val91] A. Valmari. Stubborn sets for reduced state space generation. In Proc. of 10th Int. Conf. on Applications and Theory of Petri Nets (ICATPN'90), number 483 in Lecture Notes in Computer Science. Springer, 1991. 